\title{
Proposed treatment of COVID -19 infection in poor resource setting - the use of medicinal extract from sunflower seed (Helianthus annuus seed)
}

\author{
Eli S 1, ${ }^{*}$, Orluwene CG ${ }^{2}$, Oku IY ${ }^{3}$, Bob-Manuel M ${ }^{4}$, Enyinnaya SO 4, Iyama AC 5, Nnoka VN 6 and Emeghara GI 7 \\ ${ }^{1}$ Mother, Baby and Adolescent Care Global Foundation. \\ 2 Provost College of Medical Sciences, Rivers State University/Department of Chemical Pathology University of Port \\ Harcourt Teaching Hospital. \\ ${ }^{3}$ Department of Microbiology, Niger Delta University Amassoma, Bayelsa State. \\ ${ }^{4}$ Department of Medical Microbiology and Parasitology, Rivers State University/Rivers State University Teaching \\ Hospital. \\ ${ }^{5}$ Department of Surgery, Rivers State University Teaching Hospital. \\ ${ }^{6}$ Department of Pharmacology, Rivers State University. \\ ${ }^{7}$ Department of Human Physiology, Rivers State University.
}

International Journal of Science and Research Archive, 2022, 05(01), 009-015

Publication history: Received on 21 November 2021; revised on 04 January 2022; accepted on 06 January 2022

Article DOI: https://doi.org/10.30574/ijsra.2022.5.1.0201

\begin{abstract}
Coronavirus disease (COVID 19) is an infectious disease caused by a newly discovered coronavirus. Most people infected with the COVID-19 virus will experience mild to moderate respiratory illness and recover without requiring special treatment. However, some will become seriously ill and require medical attention. In addition, older people and those with underlying medical conditions such as cardiovascular disease, diabetics, chronic respiratory disease and cancer are prone to develop severe illnesses. However, anyone can get sick with COVID 19 and become seriously ill or die at any age. With the emergence of the third wave of COVID -19 infections with a wide range of morbidities and mortalities globally, it is quite worrisome that not everyone adheres to some of these interventions especially the preventive protocols as recommended by the Centre for Disease Control (CDC).Furthermore, vaccines and some drugs alike have given a glimpse of hope in the prevention, slowing down of the progress of the disease condition and reducing the mortalities from COVID -19 infections.

Exploring the medicinal extract from Sunflower seed (Helianthus annuus seed) with myriad of medical qualities, including inherent immune properties, antioxidant, antiviral, antimicrobial, anti-inflammatory, antihypertensive, antidiabetic and wound healing properties could be a wonder drug in the treatment of COVID -19 infection with a great advantage even in pregnancy where vaccination is indicated after meticulous, evidence based research at the global level. Sunflower seed (extracts) can be stored in room temperature compared with vaccines that require cold chain system for storage.
\end{abstract}

Based on reviews of literature and proposed evaluation and treatment in poor resource settings, recommendations can be deduced from this manuscript as a follow-up for further research work on the subject the first stage of this publication.

Keywords: Proposed; Management; Covid-19; Poor resource; Sun-flower seed

\footnotetext{
${ }^{*}$ Corresponding author: Eli S

Mother, Baby and Adolescent Care Global Foundation.
}

Copyright $(2022$ Author(s) retain the copyright of this article. This article is published under the terms of the Creative Commons Attribution Liscense 4.0. 


\section{Introduction}

Coronavirus disease 2019 (COVID-19) is defined as an illness caused by a novel Coronavirus called severe acute respiratory syndrome coronavirus2 (SARS-CoV-2); formerly called 2019 nCoV), which was first identified amidanoutbreak of respiratory illness cases inWuhan City, Hubei Province, China [1].It was initially reported to the WHO on December 31, 2019. On January 30, 2020, the WHO declared the COVID-19 outbreak a global health emergency [2-4]. On March 11, 2020, the WHO declared COVID-19 a global pandemic, its first such designation since declaring HIN1influenza pandemic in 2009 [1.2].

Illness caused by SARS-CoV-2 was termed COVID-19 by the WHO, the acronym derived from "coronavirus disease". The name was chosen to avoid stigmatizing the virus's origins in terms of populations, geography, or animal associations $[5,6]$.

With the emergence of the third wave of COVID -19 infections with wide range of morbidities and mortalities globally; $7,9-11]$. it is quite worrisome that preventive measures for covid-19 infection recommended by the Centre for Disease Control (CDC) are not adhered to strictly at the local level and globally [8,12].

Furthermore, the introduction of vaccines and some drugs have given a glimpse of hope in the prevention and slowing down of the progress of the disease;[8]. and reducing the mortalities of COVID -19 infection worldwide [9].

Just like in 1820, when quinine from the back of cinchona tree was discovered and preferred treatment for resistant malaria after malaria ravaged the population of those in sub-Saharan Africa and Asia [10]. Till present day, quinine remains the main drug of choice for resistant malaria, especially for P. falciparum [10].

Exploring the medicinal extract from Sunflower seed (Helianthus annuusseed) with myriad of medical qualities, including inherent immune properties, antioxidant, antiviral, antimicrobial, anti-inflammatory, antihypertensive, antidiabetic and wound healing properties could be a wonder drug in the treatment of COVID -19 infection with a great advantage in pregnancy where vaccination is indicated after meticulous, evidence based research at the global level. Sunflower seed (extracts) can be stored in room temperature compared with vaccines that require cold chain system for storage [12-16]. Based on reviews of literature and proposed evaluation and treatment in poor resource settings, recommendations can be deduced from this manuscript as a follow-up for further research work on the subject in the first stage of this publication [2-6].

Sunflower seed contain beneficial nutrients, including healthy fats, and antioxidant components anti-inflammatory, antibacterial, prevent damage fromfree radicals, anti-cancer, anti-diabetics, anti-hypertension, and support wound healing $[1,3]$.

\section{Methods}

The published English Literature on the subject matter was obtained online (Google search engine) and in Medscape database by searching the word 'COVID -19', sunflower seed 'Helianthus annuus seed'. 'Treatment of COVID -19'. Relevant publications were reviewed and information that could be adopted for education and treatment of COVID -19 in a resource poor setting were extracted.

\section{Pathophysiology, Transmission, Diagnosis and Management of COVID-19}

\subsection{Observations}

SAR-CoV-2 is spread primarily via respiratory droplets during close face -to-face contact. Infection can be spread by asymptomatic, pre-symptomatic, and symptomatic carriers. The symptom onset is 5 days, and $97.5 \%$ of people who develop symptoms do so within 11.5 days. ${ }^{14-15}$ The most common symptoms are fever, dry cough, and shortness of breath. Radiographic and laboratory abnormalities which are lymphopenia and high lactate dehydrogenase, are common, nonspecific. ${ }^{16-20}$ Diagnosis is made by detection SAR-CoV-2via reverse transcription polymerase chain reaction (PCR) testing, although false-negative test results may occur in up to $20 \%$ to $67 \%$ of patients; however, this is dependent on the quality and timing of testing. ${ }^{4-5}$ Manifestations of COVID -19 include asymptomatic carriers and fulminant disease characterized by sepsis and acute respiratory failure. . $^{4-9,12-16}$ 
Approximately $5 \%$ of patients with COVID - 19, and $20 \%$ of those hospitalized, experience severe symptoms necessitating intensive care [1,6-9]. More than 75\% of patients hospitalized with COVID-19 require supplemental oxygen. Treatment for individuals with COVID-19 includes best practices for supportive management of acute hypoxic respiratory failure. Emerging data indicate that dexamethasone therapy reduces 28-day mortality in patients requiring supplemental oxygen compared with usual care [9,11-13]. (21.6\%vs24.6\%;age-adjusted rate ratio, 0.83 [95\%Cl,0.740.92].) and remdesivir improves time to recovery (hospital discharge or no supplemental oxygen required) from 15 to 11 days [18-23]. In a randomized trial of 103 patients with COVID-19, convalescent plasma did not shorten time to recovery. Ongoing trials are testing antiviral therapies, immune modulators, and anticoagulants [14-16]. The case fatality rate for COVID -19 varies markedly by age, ranging from 0.3 death per 1000 cases among patients 5 to 17 years to 304.9 deaths per 1000 cases among patients aged 85years or older in the US [11-14]. Among patients hospitalized in the intensive care unit, the case fatality is up to 40\% [19-20]. At least 120 SAR-CoV-2vaccines are under development [23-27]. Until an effective vaccine is available, the primary methods to reduce spread are facemasks, social distancing, and contact tracing [1-7,24-28]. Monoclonal anitbodies and hyperimmune globulin may provide additional preventive strategies $[1-4,9,29-32]$.

\subsection{Pathophysiology}

Coronavirues are large, enveloped, single-stranded RNA viruses found in humans and other mammals, such as dogs, cats, chicken, cattle, pigs and birds [1,4-9]. Coronaviruses cause respiratory, gastrointestinal and neurological disease [1-5]. The most common coronaviruses in clinical practice are 229E, OC43, NL63 and HKU1, which typically cause common cold symptoms in immunocompetent individuals [1,4-8,24-28].

SARS-CoV-2 is the third coronavirus that has caused severe disease in humans to spread globally in the past 2 decades $[1,2,25,33-35]$.

\subsection{The Host Defense Against SAR-COV-2}

Early in infection, SARS-CoV-2 targets cells, such as nasal and bronchial epithelial cells and pneumocytes; through the viral structural E2 spike(s) protein that binds to the angiotensin converting enzyme 2 (ACE2) (Figure 2). The type2 trans-membrane serine protease (TMPRSS2), present in the host cell, promotes viral uptake by cleaving ACE2 and activating the SARS-COV-2 S protein, which mediates coronavirus entry into host cells [1,2,25]. ACE2 and TMPRSS2 are expressed in host target cells, particularly alveolar epithelial type II cells similar to other respiratory viral diseases, such as influenza [1,2]. Profoundlymphopenia may occur in individuals with COVID-19 when SARS-COV-2 infects and kills T lymphocyte cells $[1,2,13]$. Addition, the viral inflammatory response, consisting of both the innate and the adaptive immune response (comprising humoral and cell-mediated immunity), impairs lymphopoiesis and increases lymphocyteapoptosis [2,12-18,25-26].

Up regulation of ACE2 receptors from ACE inhibitors and angiotensin receptor blocker medications has been hypothesized to increase susceptibility to SARS-CoV-2 infection, large observational cohorts have not found an association between these medications and risk of infection or hospital mortality due to COVID-19[227-32].

In later stages of the infection, when viral replication accelerates, epithelial barrier integrity is compromised[14-18]. In addition to epithelial cells, SARS-CoV-2 infects pulmonary capillary endothelial cells, accentuating the inflammatory response and triggering an influx of monocytes and neutrophils. The development of viral sepsis, defined as lifethreatening organ dysfunction caused by a dysregulated host response to infection, may further contribute to multiorgan failure[12-17].

\subsection{Laboratory Findings}

The typical laboratory findings in COVID-19 infections are elevated C-reactive protein (increased is $>60 \%$ in albumin of patients) lactase dehydrogenase (increased in approximately 50\% to 60\%), alanine amino-transferase (elevated in approximately 25\%), and aspartate amino transaminase (approximately 39\%)[1,10-13]. Approximately75\% have low albumen. $1,2,10$ The most common haematological abnormality is lymphopenia (absolute lymphocyte count $<1.0 \times 10^{9} / \mathrm{L}$ ), which is present in up to $83 \%$ of hospitalized patients with COVID-19 [1,2,10-12].

\subsection{Treatment of COVID-19}

The following classes of drugs are being evaluated or developed for the management of COVID-19: (e.g remdesivir, favipriravir), antibodies (e.g convalescent plasma, hyperimmune, immunoglobulins), anti-inflammatory agents (dexamethasone, statins), targeted immunomodulatory therapies (e.g tocilizumab, sarilumab, anakinra, ruxolitinib), anti-coagulants (e.g heparin),and antifibrotics (e.g tyrosine) [12-18]. It is likely that different treatment modalities 
might have different efficacies at different stages of the illness and in different manifestations of disease [1,2,10-14]. Viral inhibition would be expected to be most effective early in infection, while, in hospitalized patients, immunomodulatory agents may be useful to prevent disease progression and anticoagulates may be useful to prevent thromboembolic complications [10,18-20].

Previous studies, chloroquine /hydroxychloroquine, compounds that inhibit viral entry and endocytosis of SARS - CoV2invitro and may have beneficial immunomodulatory effects invivo, have been initiated, but early data from clinical trials in hospitalized patients with COVID-19 have not demonstrated clear benefits [12-19]. These findings do not support off label use of (hydroxy)chloroquine, either with or without the co-administration of azithromycin. Randomized clinical trials are ongoing and should provide more guidance [13-18].

Monoclonal antibodies directed against key inflammatory medications such as interferon gamma, interleukin-1 and interleukin-6, all target the overwhelming inflammatory responses following SARS - CoV-2 infections with the goal of preventing organ damage [13-19]. The interlink-6 inhibitors tocilizumab and sarilumab are best studied, with more than a dozen randomized clinical trials underway [13-18].

However, the Randomized Evaluation of COVID -19 Therapy (RECOVERY) trial, which randomized 2104 patients with COVID-19 to receive $6 \mathrm{mg}$ daily of dexamethasone for up to 10 days and to receive usual care, found that dexamethasone reduced 28 -day all-cause mortality (21.6\%vs $24.6 \%$, age adjusted rate ratio, 0.83 [ $95 \% \mathrm{Cl}, 0.74-0.92] . . \mathrm{p}<0.001)$. The benefit was greatest in patients with symptoms for more than 7 days and patients who required mechanical ventilation [8-12].

\subsection{Prognosis}

Overall hospital mortality from COVID-19 is approximately $15 \%$ to $20 \%$, but up to $40 \%$ among patients requiring ICU admission [24]. However, mortality rates vary across cohorts, reflecting the differences in the completeness of testing and case identification, variable thresholds for hospitalization, and differences in the outcome [18-22].

\subsection{Prevention and Vaccine Development}

COVID-19 is a potentially preventable disease. ${ }^{1,2,11-14}$ Because, most countries have implemented multiple infection control measures [3-5,25-31]. It is difficult to determine the relative benefit of each[12-16]. This question is increasingly important because continued interventions will be required until effective vaccines or treatments become available [1217]. Optimal measures may vary between countries based on resource limitations, geography (e.g island nations and international border measures), population, and political factors[1,2,4].

\subsection{Sunflower seed and medicinal qualities in the treatment of COVID -19 infection}

The common sunflower (Helianthus annuusL.) is a species of the Asteraceae family grown commercially worldwide offering a variety of nutritional and medicinal benefits [13-18]. The sunflower seed, although used as a snack, salad, garnish and in some bakery goods, is primarily harvested for oil production, ranking in $4^{\text {th }}$ position at world level $(8 \%$ of 186 Mt oil in 2012) after palm (29\%), soya bean (22\%) and oilseed rape (13\%) [13-16].

The sunflower seed and sprout contain valuable antioxidant, antimicrobial, anti-inflammatory, antihypertensive, wound healing and cardiovascular benefits found in its phenolic compounds, flavonoids, polyunsaturated fatty acids and vitamins [13-14].

It is used in ethnomedicine for treating a number of disease conditions including heart disease, bronchial, laryngeal and pulmonary infections, coughs and colds and the whooping cough [13-17]. These notable medicinal, nutritional and culinary benefits have resulted in historical and growing popularity of the sunflower and its constituent parts worldwide [14-19]. This review underscores the importance of increased research regarding the sunflower seed in particularly by summarizing its chemical constituents, dynamic changes, metabolic impact and overall health benefit in treatment of COVID-19 infection, taking into cognizance the antioxidant, anti-inflammatory, antiviral, anti-diabetic, antihypertensive qualities of sunflower seed [13-18].

Total cholesterol and low density lipoprotein (LDC) with antioxidant properties suppresses breast cancer gene thus prevents the development of breast cancer [14-17]. Vitamin E are considered asvital antioxidants, playing a role in preventing or controlling nonspecific reactions from various oxidizing species produced in metabolism [12-18]. 
Flavonoidsthat are isolated from sunflower with wide range of biological benefits including anti-inflammatory, antibacterial, antiviral, anti-allergic, anti-thrombotic and vasodilation activities [12-18].. These are important metabolites, found in sunflower consumed among Japanese family. ${ }^{14-18}$ The non-nutrient phytochemicals with antioxidant potentials contribute to low incidence of coronary heart diseases in Japanese women compared to women in other countries [15-19]. Isoflavine is a known phytoestrogen and has been reported to have various health beneficial roles such as anti-oxidants. The quantity of Isoflavine content in sunflower seed increase from 534ug/g in sunflower seed to 613.7 (soak in water) and $685.9 \mathrm{ng} / \mathrm{g}$ after sprouting, which indicate that sunflower sprouts may offer a better functional food source than raw sunflower seeds [14-18].

The sunflower seed is a potential treatment and preventive supplements for COVID -19 infection due to its health properties remarkable fruits, minerals, antioxidants benefits, vitamins, anti-microbial, anti-diabetic, anti-hypertensive, anti-inflammatory, and wound-healing properties [1,4-8]. In addition, these properties of sunflower seed portray advantage of pregnant women at risk of COVID -19 infection.

\section{More light on health benefits of sunflower seed in the treatment of COVID -19 infection}

\subsection{Antioxidant property}

Anti-oxidants effects have long been recognized as having protective functions against cellular damage and reduce the risk of chronic diseases [14-17]. The antioxidant activity in sunflower seedling is influenced by many factors. In the treatment of COVID -19 infections the place of antioxidants cannot be over-emphasized [1,2,10-13]. Some examples of anti-oxidants in sunflower are tocopherols - vitamin E and Phenolic acids [1,2,13-16].

\subsection{Anti-viral activity}

Research by Rauf S et al revealed that sunflower seed has medicinal components that are antiviral [32]. This makes it an important natural food that can be tapped in the treatment of COVID -19 infection. Some of these components are nicotinic acid, aldehyde, polyphenol, vanillic acid and ferullic acid [33-35].

\subsection{Improves immunity}

Sunflower seed oil contained poly unsaturated fatty acids (linoleic acid), tocopherols and phytosterols, which tends to lower low-density lipid and improve immunity against various human diseases. ${ }^{32}$ This activity of sunflower is invaluable in the treatment of COVID -19 infections especially with underlying co-morbidities like diabetes, hypertension and malignancies $[1,2,12-18]$.

\subsection{Anti-inflammatory activity}

Scholars have revealed that sunflower oil in anti-inflammatory and gastrointestinal profiles of indomethacin is evaluated in rats. ${ }^{13,15-18,22}$ These seeds also offer anti-inflammatory effect (tocopherols), hence prevent gastric damage. This medicinal anti-inflammatory characteristics may be useful in the treatment of COVID -19 patients especially as regards pneumonia and gastro-intestinal symptoms experienced by these patients [1,6-9].

\subsection{Anti-microbial activity}

In sunflower seed HA-AP10, basic polypeptide hematogens, which indicate effective anti-microbial activity against fungi [13-16]. H. annuus seed has anti-microbial activity as presented by Cortrell et al [14,25-35]. Northern specie, another specie, I helenium, another specie of sunflower seed has activity against M. tuberculosis H37rev. ${ }^{18-22}$

\subsection{Anti-diabetic}

Recent studies indicate that the sunflower seed prevents formation and accumulation of advanced glycation end products (AGES) inhyper glycemic condition is a significant contribution in diabetes control [14-16]. This will beneficial to COVID -19 patients that are diabetic [1,6-9]. Some medicinal extracts from sunflower seed with hypoglycaemic properties are vitamin A and zinc [1,2,24,35].

\subsection{Anti-hypertensive}

The sunflower seed has been shown by researchers to contain bioactive peptides which offer anti-hypertensive advantages by inhibiting the angio-tensin-1 converting enzyme (ACE1). ${ }^{18-22}$ Sunflower protein hydrolysate is obtained through hydrolysis using pepsin and pancreatin $[1,2,10]$. These peptides show different levels of ACE inhibitory for 
effectiveness at different hydrolysis times [1,4-7]. This is important in the management of COVID -19 patients that are hypertensive.

\subsection{Wound healing}

Sunflower seed/oil with a high concentration of linoleic acid can be indicated as a therapeutic alternative for both macroscopical and clinical wound healing process [18-22]. This property is of in the treatment of covid-19 patients especially by the cellular damage caused by inflammatory processes [1,2,14-18].

\section{Conclusion}

Sunflower health benefits are enormous, although under-researched. In this era of COVID -19 pandemic with the myriad of inherent potential health qualities such as anti-oxidant, anti-viral, improvement of immunity, anti-inflammatory, antimicrobial, anti-hypertensive, anti-diabetic properties. This may as well be a wonder drug in the treatment of COVID -19 infections.

\section{Compliance with ethical standards}

\section{Acknowledgments}

Mother, Baby and Adolescent Care Global Foundation.

\section{Disclosure of conflict of interest}

Authors have declared there was no conflict of interest.

\section{References}

[1] Garg S, et al. Clinical trends among US hospitalized with COVID - 19. Ann Intern Med. 2021; 10: M21-1991.

[2] Mikami T, Miyashite H, Yanid T, et al. Risk factors for mortality in patients with COVID - 19 in New York City. J GEN INTERN MED. 2021; 16:17-26.

[3] Macedo A, Gonclave SN, Febra C. COVID - 19 fatality rates in hospitalized patients: systemic review and metaanalysis. Annals of epidemiology. 2021; 57:14-21.

[4] Yen-Chin Lin. COVID-19; The first documented coronavirus pandemic in history. Biomedical Journal. 202; 43(4): $328-333$.

[5] Lone SA, Ahmad A. COVID-19 Pandemic - an African Perspective. Emerging microbes / infections. 2020; 9(11): $1300-1308$.

[6] Shereen MA, Siddique R. COVID-19 infection; Emergence, transmission, and characteristics of human coronaviruses. Journal of Advanced Research. 2020; 24: 91 - 98.

[7] Bashir T, Marshwani ZR, Zahara K, Harnder S, Mudrikah TS. Chemistry, Pharmacology and Ethno medicinal uses of Helianthus annuus (Sunflower): a review. Pure Applied Biol. 2015; 4 (2): 226 - 235.

[8] Gonzalez Perez S, Vereij Ken JM. Sunflower proteins; overview of their physiochemical, structural and functional properties. J Sci food Agric. 2007; 87(12): 2173 - 2191.

[9] Simonsen NR, Fernandez - Crehuet, Navajas J, Martia - Moreno JM. Tissue stores of individual monounsaturated fatty acids and breast cancer: the EURAMIC study on antioxidants, myocardial infarction, and breast cancer, AmericanJournalof Clinical Nutrition. 1998; 68: 134 - 141.

[10] Adam RR. Antimalarial Drug Discovery: From Quinine to the Dream of Eradication. ACS Medicinal Chemistry Letters. 2013;4(12):1126-1128.

[11] Belingheri C, Rodriguez - EsfradorMT, et al. Oxidative stability of high-oleic sunflower oil in a porous starch carrier. Food Chem. 2015; 166:346-351.

[12] Whigham LD, Watras AC, Schoeller DA. Efficacy of conjugated linoleic acid for reducing fat mass: a meta - analysis in humans; American Journal of Clinical Nutrition. 2007; 85(5):1203 - 1211. 
[13] Kamal J. Quantification of alkaloids, phenols, and flavonoids in sunflower (Helianthus annuus L.). Department of Biotechnology. 2011; 10(916): 3149 - 3151.

[14] Amakura Y, Yoshimura M, Yamakami S, Yoshida T. Isolation of phenolic constituents and characterization of antioxidant makers from sunflower (Helianthus annuus L.) seed extract. Phytochemistry Letters. 2013; 6(2): 302 -305 .

[15] Karamac M, Kosinka A, Estrella I, Hernandez T, Duenas M. Antioxidant activity of phenolic compounds indetified in sunflower seeds. European Food Research and Technology. 2012; 235(2):221-230.

[16] Fisk ID, White DA, Carvalho A, Gray DA. Tocopherol - An intrinsic component of sunflower seed oil bodies. Journal of the American Oil Chemists' Society. 2006; 83: 341-344.

[17] Kamal-Eldin A, Appelqvist LA. The chemistry and antioxidant properties of tocopherols and tocotrienol lipids. 1996;31(7):671-701.

[18] Adam KK, Lin RH. Antioxidant activity of () Agric Food Chemistry. 2002;50(21):6182-6187.

[19] Odabasoglu F, Halici Z, Cakir D, Halici M, et al. Beneficial effects of vegetable oils (corn, olive and sunflower oils) and alpha-tocopherol on anti-inflammatory and gastrointestinal profiles of indomethacin in rats. Eur J Pharmacol. 2008;591(2): 300-306.

[20] Wiersinga EJ, Rhodes A, Cheng AL, et al. Pathophysiology, Transmission, Diagnosis and Treatment of Coronavirus Disease. 2019; (COVIA-19). JAMA 202.324(8):782-793.

[21] Zaki AM, Van Boheermen S, Bestebroer TM, Osterhans AD, Fauchier RA. Isolation of a novel Coronavirus from a man with pneumonia in Saudi Arabia. N England Journal med.2012;367(19):1814-1820.

[22] Goldsmith CS, Ksiazek TG, Tatti KM et al. Ultra-structuralcharacterization of SAR Coronavirus, Emerging infectious disease. 2004;10(2):320-326.

[23] Hoffmann M, Kleine-Weber H, Schroeder S, et al. SARS-CoV-2 cell entry depends on ACE2 and TMPRSS2 and is blocked by a clinically proven protease inhibitor cell. 2020;181(20):271-280.

[24] Sungnak W, Huang N, Becavin C, et al. HCA Lung Biological Network. SARS-CoV-2 entry factors are highly expressed in nasal epithelial cells together with innate immune genes. National Med.2020;26(5):681-687.

[25] Zou X, Chen K, Zou J, Han P,Hao J, Han Z. Single-cell RNA-seq data analysis in the receptor ACE2 expression reveals the potential risk of different human organs vulnerable to 2019-nCOV infection. Front Med.2020;14(2):185-192.

[26] Xu Z, Shi L, Wang Y, et al. Pathological findings of COVID-19 associated with acute respiratory distress syndrome. Lancet Respiratory Med.2020;8(4):420-422.

[27] Thachil J, Tang N, Gado S, et al. ISTH interim guidance on recognition and management of coagulopathy in COVID19. J thrombHaemost.2020;18(5):1023-1026.

[28] Ganyani T, Kremer C, Hens N, et al. Estimating the generation interval for coronavirus disease (COVID-19) based on symptom onset data. Eurosurveill. 2020; 25(17).

[29] Leri M, Thachil J, Iba T, Levy TH. Coagulation abnormalities and thrombosis inpatients with COVID-19; Lancet Haematol.2020;7(6):4438-4440.

[30] Bai Y, Yao L, Wei T, et al. Presumed asymptomatic carrier transmission of COVID-19. JAMA. 2020;323(14):14061407.

[31] Woltel R, Corman VM, Guggemus W, et al. Virological assessment of hospitalized patients with COVID-2019. Nature. 2020;581(7809):465-469.

[32] Laver SA, Bi Q, et al. The incubation period of coronavirus disease 2019(COVID-19) from publicly reported confirm cases: estimation and application. Avin Intern Med.2020;17(9):577-582.

[33] Gang S, Kim L, Whitaker M, et al. Hospitalization rates and characteristics of patients hospitalized with laboratory confirmed coronavirus disease 2019-COVID NET, 14 states, March 1 - 30, 2020. MMWR Morb Mortal Weekly Rep. 2020;69(15):458-464.

[34] Wang W, Xu,Gad R, et al. Detection of SAR-CoV-2 in different types of clinical specimen. JAMA. 2020; 323(18):1843-1844.

[35] Rauf S, Ortiz R, Shehzad M, Haider W, Ahmed I. The exploitation of sunflower (Helianthus annuus L) seed and other parts for human nutrition, medicine and the industry". Helia. 2020;73(43): 167-184. 